\title{
Pancolitis associated with higher mortality risk of cytomegalovirus colitis in patients without inflammatory bowel disease
}

This article was published in the following Dove Press journal:

Therapeutics and Clinical Risk Management

\author{
Puo-Hsien Le ${ }^{1,2}$ \\ Chia-Jung Kuo ${ }^{1-3}$ \\ Ren-Chin $\mathrm{Wu}^{3,4}$ \\ Jun-Te Hsu ${ }^{3,5}$ \\ Ming-Yao Su ${ }^{1-3}$ \\ Chun-Jung Lin ${ }^{1,3}$ \\ Cheng-Tang Chiu ${ }^{1-3}$ \\ 'Department of Gastroenterology \\ and Hepatology, Linkou Chang Gung \\ Memorial Hospital, Taoyuan, Taiwan; \\ ${ }^{2}$ Taiwan Association for the Study of \\ Small Intestinal Diseases, Taoyuan, \\ Taiwan; ${ }^{3}$ College of Medicine, Chang \\ Gung University, Taoyuan, Taiwan; \\ ${ }^{4}$ Department of Pathology, Linkou \\ Chang Gung Memorial Hospital, \\ Taoyuan, Taiwan; ${ }^{5}$ Department of \\ General Surgery, Linkou Chang Gung \\ Memorial Hospital, Taoyuan, Taiwan
}

Correspondence: Cheng-Tang Chiu Department of Gastroenterology and Hepatology, Linkou Chang Gung Memorial Hospital, 5 Fu-Hsin Street, Queishan, Taoyuan City 333, Taiwan

Tel +8863328 I 200 ext $810 \mid$

Fax +88633272236

Email ctchiu0508@gmail.com
Background: Cytomegalovirus (CMV) colitis typically presents in immunocompromised and inflammatory bowel disease (IBD) patients. Several studies have been conducted on the endoscopic characteristics of CMV colitis in IBD patients.

Objectives: The endoscopic findings of CMV colitis in non-IBD patients and their relationship with inhospital mortality are unclear. We aimed to describe the endoscopic presentation in these patients and to determine the endoscopic predictors of inhospital mortality.

Patients and methods: Patients with CMV colitis diagnosed using histology between April 2002 and December 2016 at the Linkou Chang Gung Memorial Hospital, Taiwan, were retrospectively enrolled. Patients diagnosed with IBD during follow-up were excluded. Patient data, including underlying diseases, endoscopic presentation, laboratory data, clinical course, complications, and clinical outcomes, were collected. The independent risk factors for inhospital mortality were analyzed with logistic regression. The difference of overall survival was compared using Kaplan-Meier survival curve and log rank test. All statistical calculations were performed using SPSS software, version 21.

Results: Sixty-nine patients were enrolled, and 8 IBD patients were excluded. Within the 61 non-IBD patients, 31 were diagnosed by colonoscopy and others by sigmoidoscopy. Ulceration $(77 \%)$ was the most common endoscopic finding, followed by a cobblestone appearance (19.7\%), colitis with/without erosions (9.8\%), pseudomembrane (9.8\%), and tumor/polyp-like lesions (8.2\%). Among the patients who underwent full-length colonoscopy, 35.3\% presented with right-sided colitis, $23.5 \%$ with left-sided colitis, and $32.4 \%$ with pancolitis. Pancolitis was identified as a negative predictor of inhospital mortality (odds ratio, 6.8; 95\% confidence interval, 1.233-37.497; $p=0.028$ ) and overall survival ( $\log$ rank $p=0.018)$.

Conclusion: Colonoscopy is recommended for precise CMV colitis diagnosis and outcome prediction in non-IBD patients.

Keywords: cytomegalovirus colitis, CMV, inflammatory bowel disease, endoscopy, colonoscopy, mortality

\section{Introduction}

Cytomegalovirus (CMV) colitis is mostly diagnosed in immunocompromised and inflammatory bowel disease (IBD) patients and is associated with a high mortality. ${ }^{1-5}$ Several studies have demonstrated the endoscopic presentation of CMV colitis in IBD patients, ${ }^{6-9}$ particularly in those with ulcerative colitis, but few have done so in non-IBD patients. ${ }^{10}$ In a Korean study conducted in 2010, 43 patients were reviewed and 21 received full-length colonoscopy. ${ }^{10}$ The endoscopic findings varied, with 8 $(38 \%)$ patients displaying grossly normal mucosa in the rectosigmoid colon. However, 
the relationship between endoscopic findings and patient mortality remained unknown. We aimed to examine the endoscopic presentation of CMV colitis in non-IBD patients and to assess its relationship with mortality. In this way, we hoped to predict and decrease patient mortality in CMV colitis.

\section{Patients and methods}

\section{Compliance with ethical standards}

This study was approved by Chang Gung Medical Foundation Institutional Review Board on February 20, 2017, No 201700193B0 “Clinical presentations and outcome of cytomegalovirus infection in gastrointestinal tract" during February 1, 2017 to February 1, 2018. Informed consent from individual patients to review the medical records covering patients' data was not required for retrospective studies with the electronic medical record system, as stated by Chang Gung Medical Foundation Institutional Review Board. We confirmed patient data confidentiality. The study protocol conformed to the ethical guidelines of the 1975 Declaration of Helsinki, as reflected in a priori approval by the institution's human research committee.

\section{Patients}

Under the approval of the Chang Gung Medical Foundation Institutional Review Board (No 201700193B0), we retrospectively retrieved clinical data of all patients with pathologically proven CMV colitis from the electronic pathology database who presented at the Linkou Chang Gung Memorial Hospital between April 2002 and December 2016. Histology specimens were obtained using sigmoidoscopy or colonoscopy, and diagnosis was confirmed by the presence of CMV inclusion bodies in the tissue and a positive immunohistochemistry (IHC) staining. IHC staining was performed using monoclonal antibodies directed against the CMV pp65 antigen (Novocastra ${ }^{\mathrm{TM}}$ lyophilized mouse monoclonal antibody, Leica Biosystems Newcastle Ltd, Newcastle upon Tyne, UK).

Patients with a history of solid organ or bone marrow transplantation, HIV infection, and immunosuppressive drug use, including corticosteroids (oral or intravenous prednisone or equivalent, $\geq 20 \mathrm{mg} / \mathrm{d}$ for $>2$ weeks), or chemotherapeutic agent use within 6 months were classified as immunocompromised.

\section{Clinical and endoscopic data collection}

Clinical data collected included age; gender; dates of admission; underlying diseases; diagnosis and death, follow-up data; general condition a week prior to diagnosis; medication history; and treatment duration and outcomes. Endoscopic data collected included the distribution and appearance of colonic lesions. Laboratory data included total white blood cell and platelet counts and levels of hemoglobin, alanine aminotransferase, bilirubin, albumin, C-reactive protein and CMV pp65 antigenaemia, DNA (226-bp segment on Glycoprotein B gene, LightMix ${ }^{\circledR}$ Kit human cytomegalovirus, TIB MOLBIOL, Berlin, Germany), and serology.

\section{Statistical analysis}

Numerical data with normal distribution are presented as mean \pm standard deviation and those with nonnormal distribution are presented as medium and range. Categorical data are expressed as absolute number and percentage. We used logistic regression to identify independent risk factors for inhospital mortality, and the results are presented as odds ratio, with $95 \%$ confidence interval and $p$-value. We evaluated the overall survival using the Kaplan-Meier survival curve and $\log$ rank test. $p<0.05$ was considered statistically significant. All statistical calculations were performed using SPSS software, version 21 (IBM Corporation, Armonk, NY, USA).

\section{Results}

\section{Baseline clinicopathological characteristics}

Sixty-one non-IBD patients were enrolled. The average age was $61.2 \pm 20.0$ years, and males $(59 \%)$ were predominant. Among these, 35 (57.4\%) patients were classified as immunocompetent. Overall, 45 (73.8\%) patients received antiviral therapy, including 19 (73.1\%) immunocompromised and $26(74.3 \%)$ immunocompetent patients. In the patients with CMV antigenemia, medium of viral load was $12 / 500,000$ PMN (range 1-731/500,000 PMN). There were 4 cases with evidence of Clostridium difficile coinfection and 1 with HIV infection among them. In view of complications and outcome evaluation, $2(3.3 \%)$ patients developed bowel perforations and $6(9.8 \%)$ underwent surgical resection. The inhospital mortality was $29.5 \%$ and overall survival was $60.7 \%$. All of them died from poor general condition, especially septic shock instead of CMV colitis itself. Other data are shown in Table 1.

\section{Endoscopic findings of CMV colitis in non-IBD patients}

Thirty-one (50.8\%) patients with CMV colitis underwent full-length colonoscopy. Most patients receiving incomplete colonoscopy or sigmoidoscopy were critical, with $36.1 \%$ of 
Table I Baseline characteristics of CMV colitis patients without IBD

\begin{tabular}{|c|c|}
\hline Characteristics & Result \\
\hline Case number & 61 \\
\hline Age, years & $61.2 \pm 20.0$ \\
\hline Gender (male) & $36(59 \%)$ \\
\hline Hospital stay, day & $172.7 \pm 639.9$ \\
\hline Follow-up duration (day) & $116(3-5,178)$ \\
\hline \multicolumn{2}{|l|}{ General condition within I week } \\
\hline Sepsis & 45 (73.8\%) \\
\hline Shock & $22(36.1 \%)$ \\
\hline Respiratory failure & $22(36.1 \%)$ \\
\hline \multicolumn{2}{|l|}{ Clinical symptom triggering colonoscopy } \\
\hline Melena & $25(4 \mid \%)$ \\
\hline Diarrhea & $27(44.3 \%)$ \\
\hline Abdominal pain & $6(9.8 \%)$ \\
\hline Positive stool occult blood test & $2(3.3 \%)$ \\
\hline Other (no definite record) & $\mathrm{I}(\mathrm{I} .6 \%)$ \\
\hline Immunocompromised status & $26(42.6 \%)$ \\
\hline \multicolumn{2}{|l|}{ Underlying diseases } \\
\hline Liver cirrhosis & $4(6.6 \%)$ \\
\hline Coronary artery disease & $8(13.1 \%)$ \\
\hline End-stage renal disease & $8(13.1 \%)$ \\
\hline Diabetes mellitus & $20(32.8 \%)$ \\
\hline HIV infection & $8(13.1 \%)$ \\
\hline \multicolumn{2}{|l|}{ Immunosuppressive medication } \\
\hline Immunosuppressant & $6(9.8 \%)$ \\
\hline Chemotherapy & $\mathrm{I}(\mathrm{I} .6 \%)$ \\
\hline Steroid & $17(27.9 \%)$ \\
\hline \multicolumn{2}{|l|}{ Laboratory data } \\
\hline Total white blood cell count, $/ \mu \mathrm{L}$ & $7,800(800-27,300)$ \\
\hline Hemoglobin level, g/dL & $10.4 \pm 2.1$ \\
\hline Platelet count, $\times 1,000 / \mathrm{mm}^{3}$ & $228.0 \pm 105.8$ \\
\hline ALT, IU/L & $25.1 \pm 17.3$ \\
\hline Bilirubin, mg/dL & $0.5(0.2-7.8)$ \\
\hline Albumin, $\mathrm{g} / \mathrm{dL}$ & $2.6(1.7-4.2)$ \\
\hline C-reactive protein, $\mathrm{mg} / \mathrm{dL}$ & $73.8 \pm 76.6$ \\
\hline \multicolumn{2}{|l|}{ Viral markers } \\
\hline CMV pp65 antigenemia $(n=3 I)$ & $21(67.7 \%)$ \\
\hline CMV PCR positive $(n=7)$ & $4(57.1 \%)$ \\
\hline CMV IgG positive, IgM negative $(n=34)$ & $26(76.5 \%)$ \\
\hline CMV IgG negative, IgM positive $(n=34)$ & $0(0.0 \%)$ \\
\hline CMV IgG positive, IgM positive $(n=34)$ & $6(17.6 \%)$ \\
\hline Ganciclovir or valganciclovir treatment & $45(73.8 \%)$ \\
\hline Treatment duration, day & $19(1-151)$ \\
\hline \multicolumn{2}{|l|}{ Outcome } \\
\hline Perforation & $2(3.3 \%)$ \\
\hline Surgical resection & $6(9.8 \%)$ \\
\hline Inhospital mortality & $18(29.5 \%)$ \\
\hline Overall survival & $37(60.7 \%)$ \\
\hline
\end{tabular}

Notes: Numerical data with normal distribution are presented as mean \pm standard deviation and those with non-normal distribution are presented as medium (range). Categorical data are expressed as absolute number (percentage).

Abbreviations: ALT, alanine transaminase; CMV, cytomegalovirus; HIV, human immunodeficiency virus; IBD, inflammatory bowel disease; PCR, polymerase chain reaction.

them having shock or respiratory failure. Endoscopy showed a diverse presentation and scattered distribution (Figures 1 and 2, Table 2). When including colitis with/without erosions (45.2\%), varied ulcers were the most common presentation
(77\%). Among these ulcers, those coated with white membrane and the typical, deep ulcers (punched-out ulcers) appeared more characteristic of CMV colitis. Furthermore, 25 (40.98\%) patients had more than 1 endoscopic finding simultaneously. It was difficult to accurately diagnose or completely exclude CMV colitis using endoscopic findings without pathological confirmation.

To assess the distribution of CMV colitis, we focused on the 31 patients who underwent full-length colonoscopy. The incidence of right- and left-sided colitis and pancolitis were $35.3 \%, 23.5 \%$, and $32.4 \%$, respectively. If sigmoidoscopy was performed for CMV colitis diagnosis in nonIBD patients, $35.3 \%$ patients would be missed; therefore, colonoscopy is the preferred diagnostic tool in patients with suspected CMV colitis.

\section{Pancolitis increases mortality rate of patients with CMV colitis}

To accurately evaluate the role of endoscopic findings in inhospital mortality, we focused on 31 patients who underwent full-length colonoscopy. Among these, pancolitis was the only predictor of inhospital mortality (odds ratio, 6.8; 95\% confidence interval, 1.233-37.497; $p=0.028$ ) (Table 3). Patients with pancolitis also displayed a higher overall mortality rate (log rank $p=0.018$ ) according to the Kaplan-Meier survival curve (Figure 3). Neither immunological status nor endoscopic presentation was related to mortality. However, pancolitis was noted related to antigenemia or the reasons for endoscope.

\section{Discussion}

CMV can involve the entire gastrointestinal tract, but the colon is the most common site. ${ }^{11} \mathrm{CMV}$ colitis is mostly reported in immunocompromised and IBD patients. While pathological diagnosis is the gold standard for CMV colitis, the location and number of biopsies are also important. ${ }^{9}$ Diverse endoscopic presentations also make the endoscopic study of CMV colitis in non-IBD patients challenging.

The endoscopic findings were commonly divided into well-demarcated ulceration (50\%), ulceroinfiltrative (25\%), and pseudomembrane (25\%) types according to a study conducted on 12 patients in 2012. ${ }^{12}$ However, other case reports have shown different presentations, such as pseudotumor, longitudinal ulcers, and pseudomembranes. ${ }^{13-16}$ Although several studies have discussed the endoscopic findings of CMV colitis in IBD patients, only 1 Korean study was conducted in non-IBD patients, ${ }^{10}$ in which among 43 enrolled 

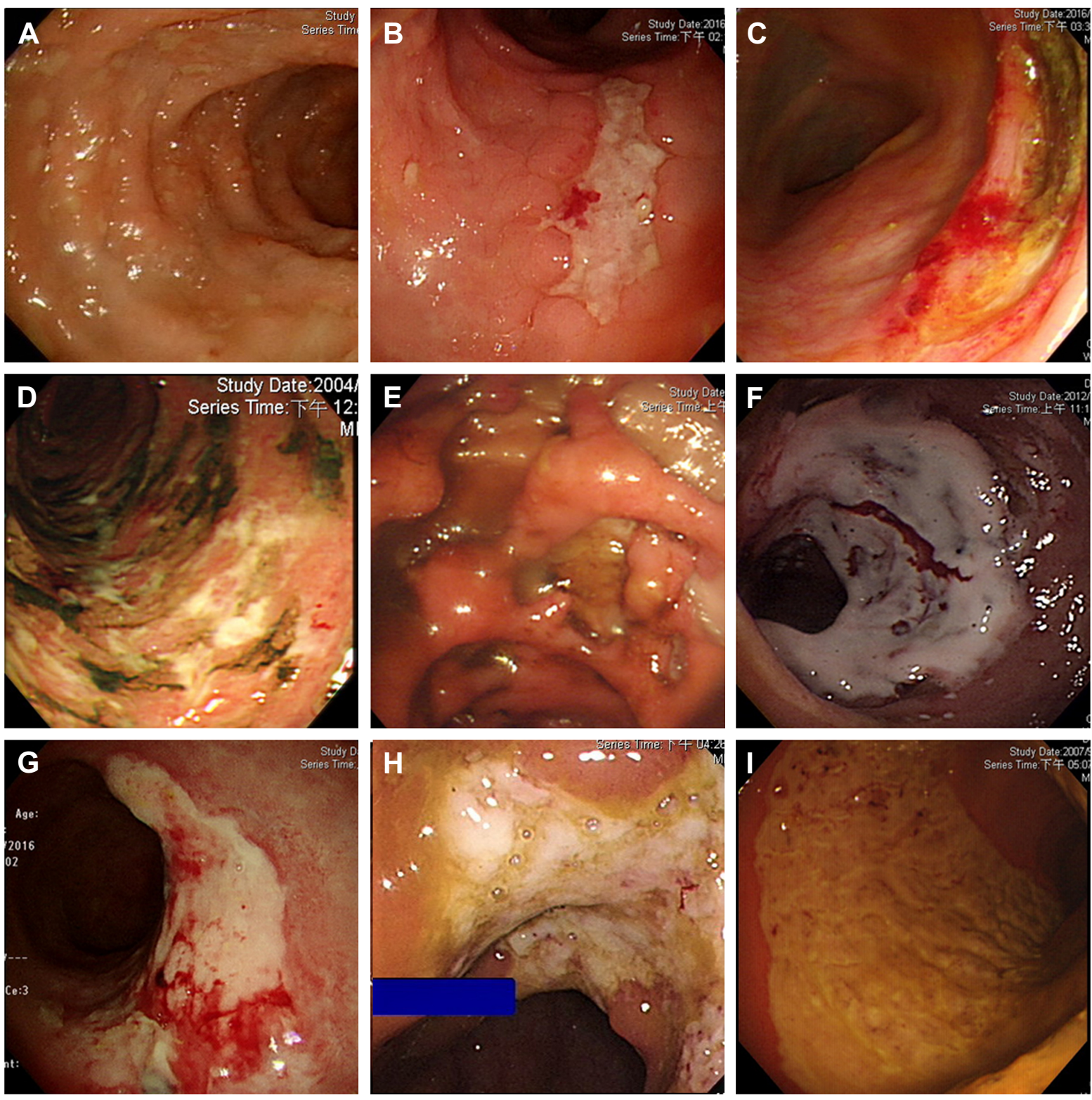

Figure I The ulcers of CMV colitis in patients without IBD.

Note: (A) Well-demarcated small ulcer, (B) longitudinal ulcer, (C) semi-lunate ulcer, (D) irregular ulcer, (E) deep ulcer (including punch-out ulcer), (F-H) ulcer coated with white membrane, and (I) segmental mucosal defect.

Abbreviations: CMV, cytomegalovirus; IBD, inflammatory bowel disease.

patients, 21 received full-length colonoscopy and presented 4 types of endoscopic findings, namely normal, colitis, ulceration, and colitis with ulceration.

In our study, we collected data from $61 \mathrm{CMV}$ colitis nonIBD patients, 31 of whom underwent full-length colonoscopy. Right-sided colitis (35.3\%) was the most common in this group of patients; therefore, a full-length colonoscopy was of greater relevance in CMV colitis diagnosis. We reported 5 major types of endoscopic findings in CMV colitis, including colitis with/without erosions, ulceration and pseudomembrane, tumor/polyp-like or cobblestone appearance. Although varied ulcers were the most common finding, only $23.4 \%$ patients presented with a typical deep or punched-out ulcer. Forty-one percent patients had at least 2 types of endoscopic findings during the diagnosis. Because of diverse endoscopic presentations of CMV colitis, we should always keep its diagnosis in mind, even in immunocompetent patients.

The inhospital mortality of CMV colitis in non-IBD patients was $29.5 \%$, and most of these patients died from poor general condition, especially septic shock rather than 

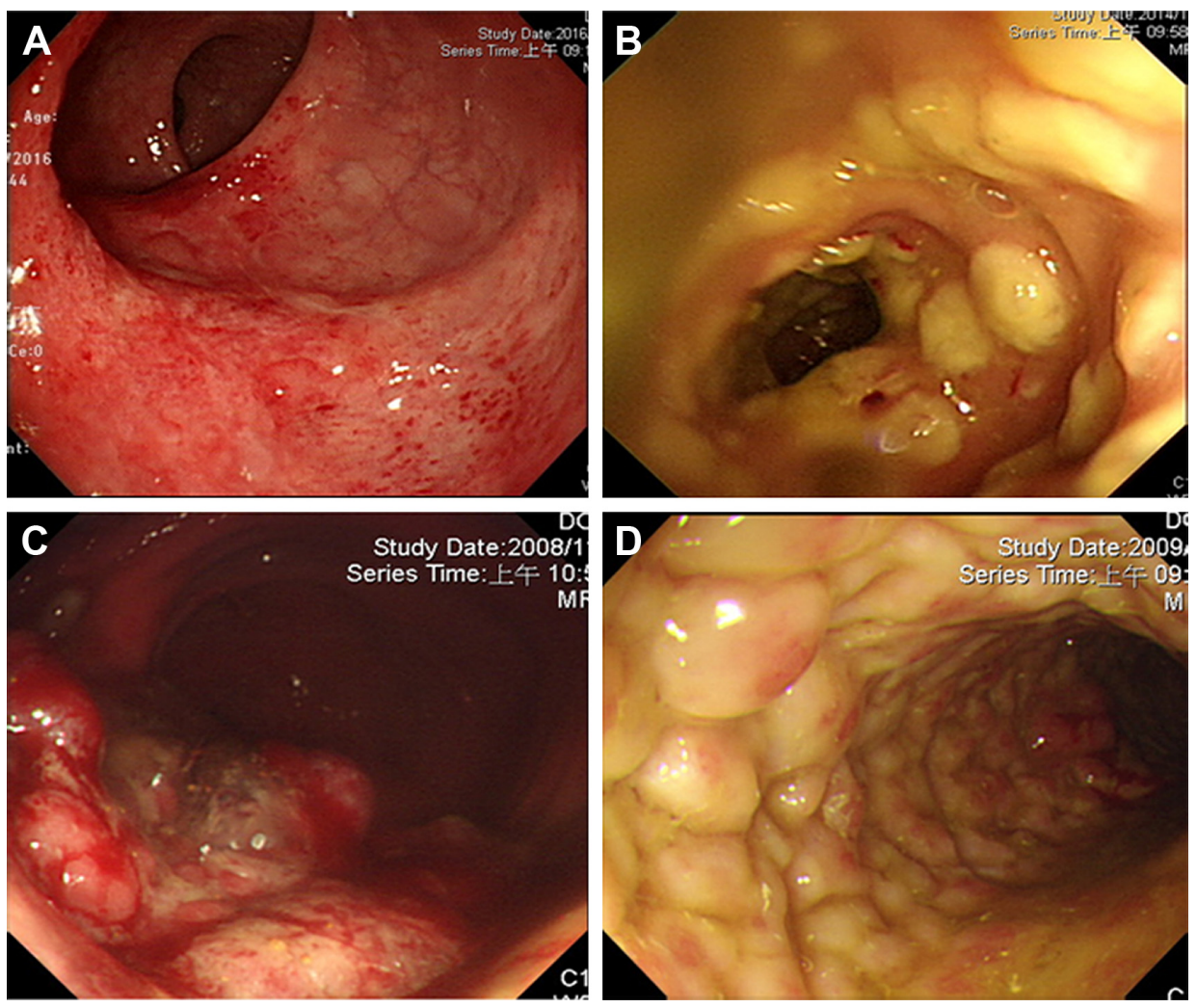

Figure 2 Endoscopic findings of CMV colitis in patients without IBD, except ulcers.

Note: (A) Colitis with/without erosion, (B) pseudomembrane, (C) tumor/polyp-like, and (D) cobblestone appearance.

Abbreviations: CMV, cytomegalovirus; IBD, inflammatory bowel disease.

Table 2 Endoscopic findings of CMV colitis patients without IBD

\begin{tabular}{ll}
\hline Characteristics & Result, $\mathbf{n}(\%)$ \\
\hline Diagnostic tools & \\
Colonoscopy & $3 I(50.8)$ \\
Sigmoidoscopy & $30(49.2)$ \\
Involved area & \\
Rectum ( $\mathrm{n}=6 \mathrm{I})$ & $35(57.4)$ \\
Sigmoid colon ( $\mathrm{n}=60)$ & $26(43.3)$ \\
Descending colon ( $\mathrm{n}=48)$ & $13(27.1)$ \\
Transverse colon $(\mathrm{n}=39)$ & $19(48.7)$ \\
Ascending colon $(\mathrm{n}=34)$ & $18(52.9)$ \\
Cecum (n=3I) & $13(41.9)$ \\
Ileocecal valve ( $\mathrm{n}=18)$ & $4(22.2)$ \\
Endoscopic finding & \\
Colitis with/without erosion, only & $6(9.8)$ \\
Ulcer & $47(77)$ \\
Well-demarcated small ulcer & $27(57.5)$ \\
Longitudinal ulcer & $5(10.6)$ \\
Semi-lunate ulcer & $21(44.7)$ \\
Irregular ulcer & $12(25.5)$ \\
Deep ulcer (including punch-out ulcer) & $1 \mathrm{I}(23.4)$ \\
Ulcer coating with white membrane & $7(14.9)$ \\
Segmental mucosal defect & $14(29.8)$ \\
Pseudomembrane & $6(9.8)$ \\
Tumor/polyp-like & $5(8.2)$ \\
Cobblestone appearance & $12(19.7)$ \\
Skip lesions & $18(29.5)$ \\
Stricture during follow-up & $2(3.3)$ \\
\hline Abrevitios &
\end{tabular}

Abbreviations: CMV, cytomegalovirus; IBD, inflammatory bowel disease. from CMV colitis itself. Pancolitis may reflect a worse clinical condition, and thus can be a negative predictor of inhospital mortality and overall survival. This is the first study to point out the relationship between endoscopic finding and mortality of CMV colitis in non-IBD patients. However, a cohort study and more cases are needed to clarify the endoscopic features.

In CMV colitis, right-sided colitis was most common, and full colonoscopy could also help us to predict the outcome. Besides, melena and diarrhea were most common presentations. Therefore, endoscopic exam was indicated in the patients with melena or diarrhea. However, critical patients had higher risks to receive full colonoscopy. In our opinion, we should advance to right side of the colon in order to completely rule out $\mathrm{CMV}$, tuberculous, or other infectious disease when we could not find active lesion over the left side of the colon in the critical patients.

\section{Conclusion}

Right-sided colitis and ulceration were the most common presentations of CMV colitis in non-IBD patients. Pancolitis 
Table 3 Clinical factors associated with inhospital mortality of CMV colitis patients without IBD (3I patients with full-length colonoscopy)

\begin{tabular}{|c|c|c|c|}
\hline Characteristics & Odd ratio & $\begin{array}{l}95 \% \text { confidence } \\
\text { interval }\end{array}$ & $p$-value \\
\hline Age & 1.036 & $0.993-1.081$ & 0.104 \\
\hline Gender (male/female) & 0.188 & $0.035-1.000$ & 0.050 \\
\hline Immunological status & 0.286 & $0.056-1.466$ & 0.133 \\
\hline \multicolumn{4}{|l|}{ Underlying diseases } \\
\hline Liver cirrhosis & 0.000 & 0.000 & 1.000 \\
\hline End-stage renal disease & $5.923 \times 10^{9}$ & $0->10^{12}$ & 0.999 \\
\hline Diabetes mellitus & 3.600 & $0.655-19.78$ & 0.141 \\
\hline HIV infection & 0.000 & 0.000 & 0.999 \\
\hline \multicolumn{4}{|l|}{ Immunosuppressive medication } \\
\hline Immunosuppressant & 2.625 & $0.146-47.183$ & 0.513 \\
\hline Chemotherapy & 4.443 & $0->10^{12}$ & 1.000 \\
\hline Steroid & 0.500 & $0.083-3.011$ & 0.449 \\
\hline \multicolumn{4}{|l|}{ Laboratory data } \\
\hline Total white blood cell count, $/ \mu \mathrm{L}$ & 1.000 & 1.000 & 0.934 \\
\hline Hemoglobin level, g/dL & 0.690 & $0.436-1.091$ & 0.112 \\
\hline Platelet count, $\times 1,000 / \mathrm{mm}^{3}$ & 0.993 & $0.984-1.003$ & 0.164 \\
\hline $\mathrm{ALT}, \mathrm{IU} / \mathrm{L}$ & 0.957 & $0.886-1.034$ & 0.267 \\
\hline Bilirubin, $\mathrm{mg} / \mathrm{dL}$ & 1.508 & $0.827-2.750$ & 0.180 \\
\hline Albumin, $g / d L$ & 0.308 & $0.055-1.736$ & 0.182 \\
\hline C-reactive protein, $\mathrm{mg} / \mathrm{dL}$ & 1.009 & $0.997-1.022$ & 0.146 \\
\hline \multicolumn{4}{|l|}{ Involved area } \\
\hline Right-sided colitis ( $n=\mid 2,35.3 \%)$ & 0.343 & $0.058-2.036$ & 0.239 \\
\hline Left-sided colitis $(n=8,23.5 \%)$ & 0.268 & $0.028-2.578$ & 0.254 \\
\hline Pancolitis (n=I I, 32.4\%) & 6.800 & I.233-37.497 & $0.028^{*}$ \\
\hline \multicolumn{4}{|l|}{ Endoscopic finding } \\
\hline \multicolumn{4}{|l|}{ Colitis with/without erosion, only } \\
\hline Ulcer & 2.353 & $0.235-23.601$ & 0.467 \\
\hline Well-demarcated small ulcer & 0.863 & $0.254-2.932$ & 0.813 \\
\hline Longitudinal ulcer & 1.667 & $0.254-10.931$ & 0.594 \\
\hline Semi-lunate ulcer & 2.051 & $0.647-6.501$ & 0.222 \\
\hline Irregular ulcer & 1.978 & $0.533-7.340$ & 0.308 \\
\hline Deep ulcer (including punch-out ulcer) & 1.469 & $0.372-5.81 \mathrm{I}$ & 0.583 \\
\hline Ulcer coating with white membrane & 3.810 & $0.757-19.172$ & 0.105 \\
\hline Segmental mucosal defect & 0.943 & $0.253-3.520$ & 0.930 \\
\hline Pseudomembrane & $4.443 \times 10^{9}$ & $0->10^{12}$ & 1.000 \\
\hline Tumor/polyp-like & 1.250 & $0.099-15.796$ & 0.863 \\
\hline Cobblestone appearance & 0.563 & $0.054-5.864$ & 0.630 \\
\hline Skip lesions & I.07| & $0.206-5.584$ & 0.935 \\
\hline Ganciclovir or valganciclovir treatment & $9.693 \times 10^{8}$ & $0->10^{12}$ & 0.999 \\
\hline Treatment duration & 0.937 & $0.830-1.057$ & 0.287 \\
\hline
\end{tabular}

Note: ${ }^{*} p<0.05$.

Abbreviations: ALT, alanine transaminase; CMV, cytomegalovirus; HIV, human immunodeficiency virus; IBD, inflammatory bowel disease.

was a negative predictor of inhospital mortality and overall survival. Colonoscopy is preferred for the diagnosis and outcome prediction of CMV colitis in non-IBD patients. The limitation of this study was small number of included cases. We will integrate more data and collect more patients to validate the conclusion in a further study.

\section{Acknowledgments}

This research received no specific grant from any funding agency in the public, commercial, or not-for-profit sectors. Prof Liang Kung-Hao, Liver Research Center, Linkou; Chang Gung of the Memorial Hospital, Taoyuan, Taiwan, provided statistical consultation. 


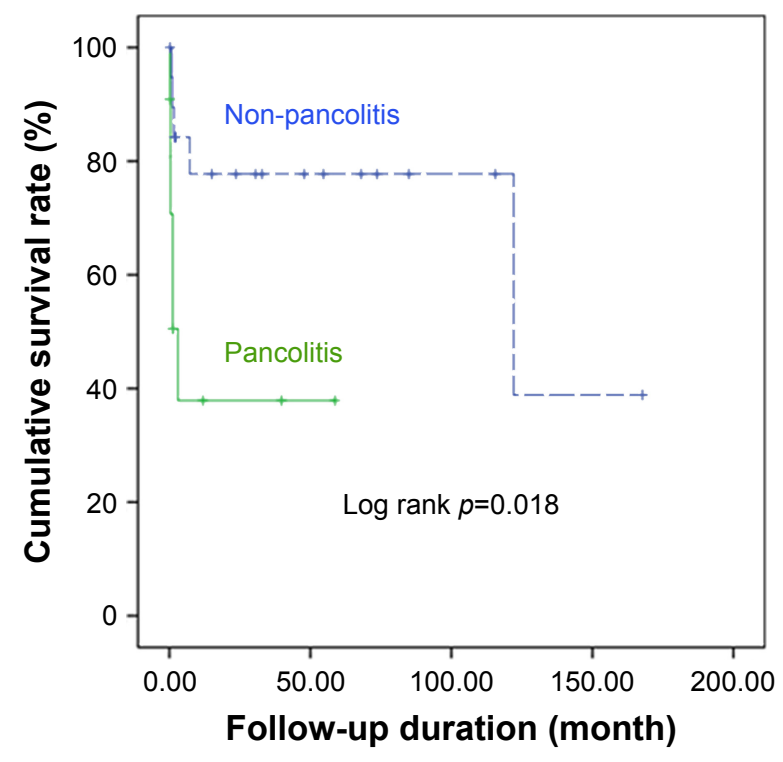

Figure 3 Survival curves of CMV colitis patients with/without pancolitis. Note: The patients of CMV colitis with pancolitis (solid line) had worse overall survival than the ones without pancolitis (dashed line) with log rank $p=0.018$ according to Kaplan-Meier survival curve analysis.

Abbreviation: CMV, cytomegalovirus.

\section{Disclosure}

The authors report no conflicts of interest in this work.

\section{References}

1. Nakase H, Herfarth H. Cytomegalovirus colitis, cytomegalovirus hepatitis and systemic cytomegalovirus infection: common features and differences. Inflamm Intest Dis. 2016;1(1):15-23.

2. Whitley RJ, Jacobson MA, Friedberg DN, et al. Guidelines for the treatment of cytomegalovirus diseases in patients with AIDS in the era of potent antiretroviral therapy: recommendations of an international panel. International AIDS Society-USA. Arch Intern Med. 1998;158(9): 957-969.

3. Matthes T, Kaiser L, Weber D, Kurt AM, Dietrich PY. Cytomegalovirus colitis - a severe complication after standard chemotherapy. Acta Oncol. 2002;41(7-8):704-706.
4. Kim YS, Kim YH, Kim JS, et al. The prevalence and efficacy of ganciclovir on steroid-refractory ulcerative colitis with cytomegalovirus infection: a prospective multicenter study. J Clin Gastroenterol. 2012;46(1):51-56.

5. Weng MT, Tung CC, Lee YS, et al. Cytomegalovirus colitis in hospitalized inflammatory bowel disease patients in Taiwan: a referral center study. BMC Gastroenterol. 2017;17(1):28.

6. Hirayama Y, Ando T, Hirooka Y, et al. Characteristic endoscopic findings and risk factors for cytomegalovirus-associated colitis in patients with active ulcerative colitis. World J Gastrointest Endosc. 2016; 8(6):301-309.

7. Suzuki H, Kato J, Kuriyama M, Hiraoka S, Kuwaki K, Yamamoto K. Specific endoscopic features of ulcerative colitis complicated by cytomegalovirus infection. World J Gastroenterol. 2010;16(10):1245-1251.

8. Yang H, Zhou W, Lv H, et al. The association between CMV viremia or endoscopic features and histopathological characteristics of CMV colitis in patients with underlying ulcerative colitis. Inflamm Bowel Dis. 2017;23(5):814-821.

9. McCurdy JD, Enders FT, Jones A, et al. Detection of cytomegalovirus in patients with inflammatory bowel disease: where to biopsy and how many biopsies? Inflamm Bowel Dis. 2015;21(12):2833-2838.

10. Kim CH, Bahng S, Kang KJ, et al. Cytomegalovirus colitis in patients without inflammatory bowel disease: a single center study. Scand J Gastroenterol. 2010;45(11):1295-1301.

11. Galiatsatos P, Shrier I, Lamoureux E, Szilagyi A. Meta-analysis of outcome of cytomegalovirus colitis in immunocompetent hosts. Dig Dis Sci. 2005;50(4):609-616.

12. Seo TH, Kim JH, Ko SY, et al. Cytomegalovirus colitis in immunocompetent patients: a clinical and endoscopic study. Hepatogastroenterology. 2012;59(119):2137-2141.

13. Inoue K, Wakabayashi N, Fukumoto K, et al. Toxic megacolon associated with cytomegalovirus infection in a patient with steroid-naive ulcerative colitis. Intern Med. 2012;51(19):2739-2743.

14. Vegunta AS, Dasar SK, Joshi SK, Rao RV. Spontaneous partial vanishing cytomegalovirus pseudotumour of colon in an immunocompetent patient. J Clin Diagn Res. 2015;9(8):TD07-TD09.

15. Falagas ME, Griffiths J, Prekezes J, Worthington M. Cytomegalovirus colitis mimicking colon carcinoma in an HIV-negative patient with chronic renal failure. Am J Gastroenterol. 1996;91(1):168-169.

16. Roskell DE, Hyde GM, Campbell AP, Jewell DP, Gray W. HIV associated cytomegalovirus colitis as a mimic of inflammatory bowel disease. Gut. 1995;37(1):148-150.
Therapeutics and Clinical Risk Management

\section{Publish your work in this journal}

Therapeutics and Clinical Risk Management is an international, peerreviewed journal of clinical therapeutics and risk management, focusing on concise rapid reporting of clinical studies in all therapeutic areas, outcomes, safety, and programs for the effective, safe, and sustained use of medicines. This journal is indexed on PubMed Central, CAS,

\section{Dovepress}

EMBase, Scopus and the Elsevier Bibliographic databases. The manuscript management system is completely online and includes a very quick and fair peer-review system, which is all easy to use. Visit http://www.dovepress.com/testimonials.php to read real quotes from published authors. 